\title{
Regularized Kalb-Ramond magnetic monopole with finite energy
}

\author{
Nick E Mavromatos and Sarben Sarkar \\ Theoretical Particle Physics and Cosmology Group, Department of Physics, King's College London, \\ Strand, London WC2R 2LS, United Kingdom
}

(Received 5 April 2018; published 13 June 2018)

\begin{abstract}
In a previous work we suggested a self-gravitating electromagnetic monopole solution in a stringinspired model involving global spontaneous breaking of a $\mathrm{SO}(3)$ internal symmetry and Kalb-Ramond (KR) axions, stemming from an antisymmetric tensor field in the massless string multiplet. These axions carry a charge, which, in our model, also plays the role of the magnetic charge. The resulting geometry is close to that of a Reissner-Nordström (RN) black hole with charge proportional to the KR-axion charge. We proposed the existence of a thin shell structure surrounding a (large inner) core as the dominant mass contribution to the energy functional. Although the resulting energy was finite, and proportional to the KRaxion charge, the size of the shell was not determined and left as a phenomenological parameter. In the current article, we propose a new way to calculate the size of the thin shell: string theory considerations suggest that the short-distance physics inside the inner core may be dominated by a positive cosmological constant term proportional to the scale of the spontaneous symmetry breaking of $\mathrm{SO}(3)$. The size of the shell is estimated by matching the RN metric of the shell to the de Sitter metric inside the core. The matching entails the Israel junction conditions for the metric and its first derivatives at the inner boundary of the shell, and determines the inner mass-shell radius. The axion charge plays an important role in guaranteeing the positivity of the "mass coefficient" of the gravitational potential term appearing in the metric; so, the KR electromagnetic monopole shows normal attractive gravitational effects. This is to be contrasted with the axion-less global monopole case where such a matching is known to yield a negative "mass coefficient" (and, hence, a repulsive gravitational effect). The total energy of our monopole within the shell is calculated. As a result of the violation of Birkhoff's theorem (due to the formal divergence of the energy functional in the absence of a large distance cutoff), the total energy does not have to equal the mass coefficient. However, for phenomenologically relevant sets of parameters, the ratio of the total energy and the mass coefficient in the shell is close to 1 . The gravitational "effective mass coefficient" in the shell can be made equal to the total energy outside the core by a small decrease in the cosmological constant in the de Sitter region. This is achieved through a dilaton potential which is suitably negative inside the de-Sitter region, but vanishes outside that region.
\end{abstract}

DOI: 10.1103/PhysRevD.97.125010

\section{INTRODUCTION: A REVIEW OF THE MODEL}

Recently [1] the authors have formulated a novel magnetic monopole solution in a string-inspired model, involving the coupling of self-interacting scalar fields, responsible for the spontaneous breaking of a global SO (3) symmetry, to a Kalb-Ramond (KR) field, associated with the spin-one antisymmetric tensor field of the string gravitational multiplet. The model generalizes the gravitational monopole model [2] and contains, in addition, electromagnetic $U(1)$ gauge fields, which couple to the

Published by the American Physical Society under the terms of the Creative Commons Attribution 4.0 International license. Further distribution of this work must maintain attribution to the author(s) and the published article's title, journal citation, and DOI. Funded by SCOAP ${ }^{3}$.
KR field via a dilaton field - the dilaton is the scalar field of the string gravitational multiplet.

The low energy effective Lagrangian ${ }^{1}$ of the model is

$$
L=(-g)^{1 / 2}\left\{\begin{array}{l}
\frac{1}{2} \partial_{\mu} \chi^{A} \partial^{\mu} \chi^{A}-\frac{\lambda}{4}\left(\chi^{A} \chi^{A}-\eta^{2}\right)^{2}-R \\
+\frac{1}{2} \partial_{\mu} \Phi \partial^{\mu} \Phi-V(\Phi) \\
-\frac{1}{12} e^{-2 \Phi} H_{\rho \mu \nu} H^{\rho \mu \nu}-\frac{1}{4} e^{-\Phi} f_{\mu \nu} f^{\mu \nu}
\end{array}\right\} .
$$

In the above expression, $-g$ denotes the determinant of the metric, $\chi^{A} ; A=1,2,3$, are the scalar fields responsible for the spontaneous breaking of the $\mathrm{SO}(3)$ internal symmetry via their vacuum expectation value (v.e.v.) $\eta$; $H_{\mu \nu \rho}=\partial_{[\mu} B_{\nu \rho]}$ is the totally antisymmetric field strength of

\footnotetext{
${ }^{1}$ The Lagrangian is inspired by perturbative weakly coupled string theory.
} 
the antisymmetric tensor field $B_{\mu \nu}=-B_{\nu \mu}$ (with $[\ldots]$ denoting antisymmetrization of the respective indices); $f_{\mu \nu}$ is the Maxwell tensor of the electromagnetic $U(1)$ gauge field; $\Phi$, the dilaton field, is stabilized (in perturbative string theory) to a constant value $\Phi=\Phi_{0}$ at the minimum of $V(\Phi)$ with $V\left(\Phi_{0}\right)=0$. For a core region surrounding the monopole center where gravity is strong, the underlying string theory may be strongly coupled. In this core region the behavior of the dilaton may result in a regularization of the associated space-time singularity [1] of the monopole. This regularization and its ramification is the subject of this paper.

For the lowest order string effective action [3,4] in terms of the gravitational multiplet, it is known that the field strength $H_{\mu \nu \rho}$ plays the role of a totally antisymmetric torsion in a generalized Christoffel connection. In four space-time dimensions, the dual of the torsion is a pseudoscalar (KR axion) field $b(x)$ [5],

$$
H_{\mu \nu \rho}=\epsilon_{\mu \nu \rho \sigma} e^{2 \Phi} \partial^{\sigma} b(x)
$$

with $\epsilon_{\mu \nu \rho \sigma}$ the covariant Levi-Civita antisymmetric symbol. As discussed in [1], the classical radial solution for $b(r)$ reads $^{2}$ :

$$
b^{\prime}(r)=\frac{\zeta}{r^{2}} \sqrt{\frac{A(r)}{B(r)}}
$$

where $r$ is the radial distance (with dimension of length) from the centre of the configuration. The quantity $\zeta$ is a dimensionless constant of integration which measures the strength of the KR field strength and the pseudoscalar field $b(x)$ has mass dimension one. We call $\zeta$ the KR-axion charge.

The (dimensionless) quantities $A(r)$ and $B(r)$ are radial functions appearing in the solution for the metric tensor [1]

$$
g_{\mu \nu}=\left(\begin{array}{cccc}
B(r) & & & \\
& -A(r) & & \\
& & -r^{2} & \\
& & & -r^{2} \sin ^{2} \theta
\end{array}\right) .
$$

\footnotetext{
${ }^{2}$ In [1] we fixed the dilaton to a constant background $\Phi_{0}$ such that the string coupling $g_{s}=e^{\Phi_{0}}=\frac{1}{\sqrt{2}}$, which is compatible with the order of magnitude of string couplings $\frac{g_{s}^{2}}{4 \pi}=\frac{1}{20}$ characteristic of phenomenologically relevant models,. We also absorbed the factor $e^{-\Phi_{0} / 2}$ in a redefinition of the electromagnetic field strength $f_{\mu \nu}$, so that one has a canonically normalized Maxwell term in (1). It is with these conventions that the normalization of the axion charge $\zeta$ in (3) is fixed. An arbitrary constant value of $\Phi_{0}$ can thus be absorbed in an appropriate normalization of $\zeta$.
}

We have from [1]: $A(r) B(r)=1+\mathcal{O}\left(r^{2}\right)$, for $r \rightarrow 0$, while $A(r) B(r)=1+\mathcal{O}\left(r^{-2}\right)$ for $r \rightarrow \infty$. In the current work we shall adopt the following approximate relations for the entire range of $r$

$$
\begin{aligned}
A(r) B(r) & \approx 1, \\
B(r) & =1-8 \pi \mathrm{G} \eta^{2}-\frac{2 m \mathrm{G}}{r}+\frac{8 \pi \mathrm{G} p}{r^{2}},
\end{aligned}
$$

where $m$ is the Schwarzschild mass of the monopole, $r$ is the radial distance from the centre, both having the appropriate dimensions and $G$ is Newton's gravitational constant of four dimensional space time. For the solution [1] of phenomenological relevance to current colliders (i.e., with a detectable monopole mass): if $8 \pi \mathrm{G} \eta^{2} \ll 1$, with $\eta$ assumed to be much lower than the Planck scale, the monopole might have a mass $\mathcal{O}(10 \mathrm{TeV})$. The solution (5) is the Reissiner-Nordström ( $\mathrm{RN}$ ) expression [6] for a magnetic black hole and is compatible with the asymptotic forms studied in [1] and so the expression (5) provides a good approximation for our purposes. From (3) and (5) we obtain the following solution for the KR axion

$$
b^{\prime}(r)=\frac{\zeta}{r^{2} B(r)}
$$

It will be convenient to use dimensionless variables, and so we will now work in units of $8 \pi \mathrm{G}=1$. In these units the metric function $B(r)$ (5) become:

$$
\begin{aligned}
B(r) & =1-\eta^{2}-\frac{2 M}{r}+\frac{p}{r^{2}} \\
p & =2 \zeta^{2}, \quad M \equiv \frac{m}{8 \pi}, \quad r \rightarrow \frac{r}{\sqrt{8 \pi}},
\end{aligned}
$$

with $r, M$ and $\eta$ dimensionless (or, equivalently, expressed in reduced Planck mass scale units, in which the Planck mass is $M_{P}=\ell_{P}^{-1}=\sqrt{8 \pi}$, with $\ell_{P}$ the Planck length).

As discussed in detail in [1], the solution for the electromagnetic $U(1)$ Maxwell tensor is [1]:

$$
f_{\mu \nu}=\left(\begin{array}{cccc}
0 & 0 & 0 & 0 \\
0 & 0 & 0 & 0 \\
0 & 0 & 0 & 2 r \sin \theta W(r) \\
0 & 0 & -2 r \sin \theta W(r) & 0
\end{array}\right),
$$

with

$$
W(r)=\frac{\zeta}{r}
$$

for all $r$. 
The associated magnetic field has only a radial component, which in contravariant form reads [1]:

$$
\mathcal{B}^{r}=\frac{1}{\sqrt{-g}} \eta^{r \theta \phi} f_{\theta \phi}=\sqrt{\frac{2}{A B}} \frac{W(r)}{r} \simeq \frac{\sqrt{2} \zeta}{r^{2}},
$$

where we took into account Eqs. (4) and (5). From this it follows that the magnetic charge is $g= \pm \sqrt{2} \varsigma$. The electric field and charge are zero. It is evident from (5) that the constant $p$ is the square of the magnetic charge,

$$
p=g^{2}=2 \zeta^{2}
$$

and thus the KR-axion charge provides the (Dirac) magnetic charge in this model [1], via the RN geometry (5) $[(7)]$,

$$
g=\sqrt{2} \zeta
$$

Dirac quantization then leads to large values of the KR axion charge $\zeta$ (in our natural units), since the Dirac quantization condition is

$$
g e=\frac{n}{2}, \quad n \in \mathbf{Z}^{+} .
$$

The global gravitational monopole is known to modify the four-dimensional asymptotic space-time to that of a curved space-time with a conical singularity, corresponding to a deficit angle $8 \pi \mathrm{G} \eta^{2}$ (cf. (5) with scalar curvature

$$
R \propto \frac{16 \pi \mathrm{G} \eta^{2}}{r^{2}}
$$

Similar features remain for the RN geometry in [1].

For the scalar triplet field, associated with the spontaneous symmetry breaking of the global $\mathrm{SO}(3)$ symmetry, we made the ansatz [1]

$$
\chi^{A}(r)=\eta f(r) \frac{x^{A}}{r}, \quad A=1,2,3,
$$

where $x^{A}, A=1,2,3$ are Cartesian spatial coordinates, with the asymptotic behavior

$$
\begin{aligned}
f(r \rightarrow 0) & \simeq f_{0} r \rightarrow 0 \quad f_{0}=\text { constant } \in \mathrm{R}, \\
f(r \rightarrow \infty) & \rightarrow 1 .
\end{aligned}
$$

In [1] we adopted a heuristic approach to demonstrating the finiteness of the monopole mass, based on the assumption of a bag-like structure. Specifically, we assumed that the entirety of the mass resides inside a bag of radius $L_{c}$, and, in fact, within a thin shell bounded by the (large) shell radius, yielding the following estimate for the total energy of the magnetic monopole [1]:

$$
\mathcal{E} \simeq 4 \pi \int_{\alpha L_{c}}^{L_{c}} d r r^{2}\left[\begin{array}{l}
\frac{2 W(r)^{2}}{B(r) r^{2}}+\frac{b^{\prime}(r)^{2}}{4}+ \\
\eta^{2}\left(\frac{f(r)^{2}}{B(r) r^{2}}+\frac{f^{\prime}(r)^{2}}{2}\right)+ \\
\frac{\lambda \eta^{4}}{4 B(r)}\left(f(r)^{2}-1\right)^{2}
\end{array}\right],
$$

where $0<\alpha<1$ is a dimensionless phenomenological parameter determining the mass shell thickness. Inside the shell, there are non-trivial configurations of the KR axion, electromagnetic field, and the scalar triplet fields $\chi^{A}, A=1$, 2, 3 responsible for the $\mathrm{SO}(3)$ spontaneous symmetry breaking in the model. Upon the assumption of large $L_{c}$ and $\alpha L_{c}$ (as compared to the Planck length), one obtains from (17)

$$
\begin{aligned}
& \mathcal{E} \simeq \frac{1}{\alpha}(1-\alpha)\left(9 \pi \zeta^{2}-\frac{4 \pi}{\lambda}\right) \frac{1}{L_{c}}+4 \pi \eta^{2}(1-\alpha) L_{c}, \\
& L_{c}, \quad \alpha L_{c} \gg 1 .
\end{aligned}
$$

In arriving at the above result we used (9), as well as the asymptotic behavior of the scalar triplet $\chi^{A}, A=1$, 2, 3, for $\lambda \eta^{2} r \gg 1$, i.e., $f(r) \simeq 1-\frac{2}{\lambda \eta^{2} r^{2}}$, found in [1]. As in [1] we will ignore the $1 / \lambda$ terms in (18) since we are working in the large $\lambda \gg 1$ limit; we note the relative minus sign of this term relative to the $\zeta^{2}$ term in (18). (This observation also corrects a typographical error in the corresponding formula for the total monopole energy in [1].)

We have assumed (as in [1]) that the solution (9) for the electromagnetic field is valid for both small and large $r$. This follows from the dilaton equation of motion of the original Lagrangian (1) of the model [1], which requires that the (covariant) square of the electromagnetic tensor $f_{\mu \nu} f^{\mu \nu}$ is proportional to the KR kinetic term $\partial_{\mu} b \partial^{\mu} b$. Since the solution for the $b$-field (3) is valid uniformly for $r$ (both small and large $r$, compared to the Planck length), the kinetic term for $b$ is $\zeta^{2} / r^{4}$ in leading order for the two limits; the ansatz (8) then implies the validity of (9) for both large and small $r$. It should also be remarked that the metric in [1] is not rigorously $\mathrm{RN}$, since the product $A(r) B(r)$ is not exactly 1 for all $r$, but resembles a RN space-time to a very good approximation in the limit of both large and small $r$. Moreover, these two asymptotic RN space-times are characterized by charge parameters, which differ from each other by terms of order $1 / \lambda$; thus only in the limit $\lambda \rightarrow \infty$ does one 
obtain the same RN metric in the $r \rightarrow 0$ and $r \rightarrow \infty$ limits [1]. In view of the small- $r$ regularization of the KR self-gravitating monopole solution (using de Sitter spacetime in a core region) to be discussed in the next Sec. II, we will only be interested in the large $r$ limit. The radius $\delta$ of the core region will turn out to be much larger than the Planck length scale but, in a string theory with large extra dimensions, it can be of order of the string scale $[3,4]$.

The assumption of a single $\mathrm{RN}$ space-time outside the de Sitter region is valid for all $\lambda$. We assumed in [1] strong coupling $\lambda \gg 1$ in order to ensure that the scalar triplet fields are near their vacuum expectation value, i.e., large quantum fluctuations are suppressed in the respective path integral; we obtain $L_{c}$ by minimizing the right-hand side of (18) with respect to $L_{c}$ :

$$
L_{c} \simeq \frac{3}{2} \sqrt{\frac{1}{\alpha}} \frac{|\zeta|}{\eta}
$$

which implies

$$
\mathcal{E} \simeq 12 \pi \sqrt{\frac{1}{\alpha}}(1-\alpha)|\zeta| \eta
$$

In [1] the parameter $\alpha$ could not be estimated. In general, $\sqrt{\alpha}$, and hence the size of the shell, could depend on the coupling $\lambda$, but it was the assumption in [1] that any potential $\lambda$-dependence in $\alpha$ was such that the terms of order $\mathcal{O}\left(\frac{1}{\lambda}\right)$ [that were ignored in deriving (19) and (20)] were subleading.

It is the purpose of this note to attempt a resolution of these important issues by demonstrating in detail the emergence of such baglike shell structures in our selfgravitating monopole solution on implementing a string theory inspired regularization of the physical singularity at $r \rightarrow 0$ (14). This regularization allows us to calculate the finite monopole mass in terms of the parameters of the model (1), by providing a natural estimate of the parameter $\alpha$.

\section{REGULARIZING THE CURVATURE SINGULARITY OF THE KR ELECTROMAGNETIC MONOPOLE}

For a self-gravitating KR electromagnetic monopole [1], the metric assumes the static RN form (4), (5), with an angular deficit proportional to $\eta^{2}$. We may regularize the associated curvature singularity (14) by considering a (small) region around the singularity and replacing the space-time inside it by an appropriate space-time of de Sitter type of radius $\delta$. (See Fig. 1, where the white (dark) shaded region corresponds to the de Sitter (RN with angular deficit) space-times.). The outer space-time corresponds to the $\mathrm{RN}$ metric (4). ${ }^{3}$ The cutoff radius $\delta$ of the appropriate boundary that separates the two space times may be determined by employing the well-known procedure initiated by Israel [7] based on the matching of two spherical regions in space, an inner and an outer one, described by different metrics. The regions are separated by a "thin-shell." We assume, following [8], that the energymomentum tensor vanishes on this interface hyperplane, and so no energy flow occurs through the boundary surface at $r=\delta$.

The matching conditions ("Israel conditions") amount to demanding the continuity of the metric and its derivatives on the thin shell. This implies continuity of the curvature and thus (in view of the Einstein equations) the stressenergy tensor of the model. This procedure was applied to a regularization of the self-gravitating global monopole of [2] in [9], and for the conventional RN metric in $[8,10]$. (In [10] it was also argued that, for (restricted) stability, the

\footnotetext{
${ }^{3}$ In the context of our string/brane-theory inspired model, with its low-energy Lagrangian (1), this regularization may be understood as follows: the regularization concerns the ultraviolet region near the centre of the black hole/monopole, $r \rightarrow 0$, where gravity is strong and so a strongly coupled string theory might be expected, with a string coupling $g_{s}(r \rightarrow 0)=e^{\Phi(r \rightarrow 0)} \gg 1$. Consequently, a nonconstant nature of the dilaton, ignored in our analysis outside the core region, becomes important. From the effective Lagrangian (1) we note that the dilaton does not couple directly to the terms involving the scalar triplet fields $\chi^{a}, a=1$, 2,3 . As a result, although the scalar fields go to zero as $r \rightarrow 0$, the contribution of the term $\frac{1}{4} \lambda \eta^{4}$ from the scalar potential remains, and is independent of the dilaton. The antisymmetric tensor and electromagnetic terms, on the other hand, are suppressed due to the dilaton prefactors (which are inversely proportional to powers of the string coupling). This is implied by (1) on using the dilaton equation of motion and requiring that, in the strong coupling limit of string theory, the field strength $H_{\mu \nu \rho}$ (2) remains finite. Furthermore, in the de Sitter region, higher-order derivative terms in the string effective action become important, whose form leads for to Born-Infeld type electromagnetism [3]. Both the dilaton prefactors and the Born-Infeld form of the electromagnetic terms imply a subdominant role compared to that of the $\frac{1}{4} \lambda \eta^{4}$ in the effective action (see Ref. [4] for more details). One might expect that strong string couplings characterize length scales of order of the string length, and indeed the radius of the de Sitterdomination region $\delta$ may be identified with the corresponding string length scale $\ell_{s}=1 / M_{s}$, where $M_{s}$ is the corresponding string mass scale. As we shall see later on, the scale $\delta$ is much larger than the four-dimensional Planck length, which implies a large string length scale. However, the above arguments are rather heuristic and valid only within the context of our low energy effective Lagrangian (1), which describes the tree-level dynamics of an underlying string theory model. Hence they do not constitute a "derivation" of our regularization procedure in the context of a microscopic string theory model. For strong string couplings, string loop corrections become important, and the nonperturbative action is not known in closed form. However, for our purposes of regularizing the black hole singularity, such heuristic constructions suffice to give a plausible justification for using de Sitter space as a way of regularizing the black-hole singularity in our string-inspired field theory model.
} 


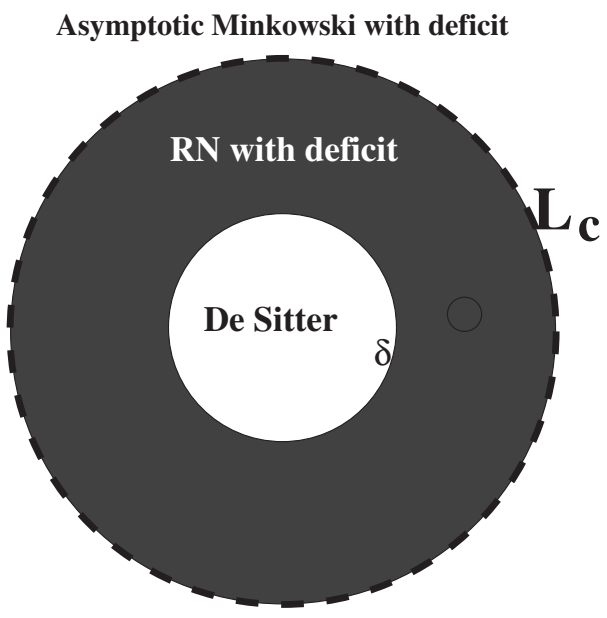

FIG. 1. The regularized self-gravitating KR electromagnetic monopole. Israel matching conditions apply at the inner shell radius $\delta$ between the inner-shell de Sitter space time and the exterior RN space time with an angular deficit proportional to the $\mathrm{SO}(3)$ spontaneous symmetry breaking scale $\eta^{2}$. The dark shaded area depicts the area where most of the mass of the monopole lies. The outer radius of the shaded boundary, $L_{c}$, is sufficiently large (compared to the Planck length), so that, at this boundary, the RN space time with the angular deficit $\propto \eta^{2}$ is, to a very good approximation, an asymptotic Minkowski space time with the same angular deficit. Hence no additional matching is required at the core radius $L_{c}$, as the $\mathrm{RN}$ space extends formally from $\delta$ to infinity. This is indicated by the dashed-line outer boundary (at $L_{c}$ ) of the dark shaded region.

perturbed shell has to satisfy a certain polytropic equation of state). For a different approach to the regularization of black-hole singularities see [11].

\section{A. Israel conditions for regularized KR monopole}

In our baglike model for the KR magnetic monopole [1], the outer core radius $L_{c}$ is determined by minimization of the energy functional integrated over the shell region between the radius $\delta$ (to be identified with the lower integration limit $\alpha L_{c}$ ) and $L_{c}$ in (17). The radius $L_{c}$ is assumed to be sufficiently large to act as an upper spatial cutoff for the otherwise divergent energy of the monopole. This divergence (which is shared with the global monopole case of [2]) is due to the existence of the deficit $\eta^{2}$ in the induced space-time (which differentiates it from the standard Minkowski space-time). No matching conditions are required on the outer core surface of radius $L_{c} \gg 1$, since the RN space-time with deficit extends formally to infinity.

In terms of spherical polar coordinates $r, \theta, \varphi$ we consider the following infinitesimal line element for the regularized space-time:

$$
d s_{\mathrm{reg}}^{2}=f(r) d t^{2}-\frac{d r^{2}}{f(r)}-r^{2}\left(d \theta^{2}+\sin ^{2} \theta d \varphi^{2}\right),
$$

where the distribution function $f(r ; \delta)$ is defined as follows:

$$
\begin{aligned}
f(r ; \delta) & =B_{1}(r) \Theta(\delta-r)+B(r) \Theta(r-\delta), \\
B_{1}(r) & =1-\frac{1}{3} \Lambda r^{2}, \quad \Lambda>0,
\end{aligned}
$$

with $B(r)$ given in (7) and the positive parameter $\Lambda$ will be chosen later. In our static case, the Israel matching conditions are:

$$
\begin{aligned}
B_{1}(r=\delta) & =B(r=\delta), \\
\left.\frac{d}{d r} B_{1}(r)\right|_{r=\delta} & =\left.\frac{d}{d r} B(r)\right|_{r=\delta},
\end{aligned}
$$

which imply

$$
\begin{aligned}
\frac{\Lambda}{3} & =\eta^{2}+\frac{2 M}{\delta}-\frac{2 \zeta^{2}}{\delta^{2}}, \\
-\frac{2}{3} \Lambda \delta & =\frac{2 M}{\delta^{2}}-\frac{4 \zeta^{2}}{\delta^{3}} .
\end{aligned}
$$

These determine the cutoff radius $\delta>0$ and the "mass coefficient" $M$ of the metric (7) in terms of the de Sitter parameter $\Lambda>0^{4}$ :

$$
\begin{aligned}
\delta & =\frac{\eta}{\sqrt{2 \Lambda}}\left(1+\sqrt{1+\frac{8 \zeta^{2} \Lambda}{\eta^{4}}}\right)^{1 / 2}>0, \\
2 M & =-\frac{2}{3} \Lambda \delta^{3}+\frac{4 \zeta^{2}}{\delta} .
\end{aligned}
$$

We note from (25) that in the absence of the KR-axion charge (i.e., $\zeta=0$, the global monopole case [2]), the mass coefficient $M=-\frac{2 \Lambda}{3} \delta^{3}<0$ is negative; since this coefficient appears in the gravitational potential, this case implies repulsive gravitational effects of the global monopole, as discussed in [9]. The situation changes drastically for the KR electromagnetic monopole case [1]; since, as can be deduced from (25), in the presence of sufficiently large $\zeta^{2}$, [necessary for the Dirac quantization condition (13)], strong coupling and appropriate values of $\Lambda>0$ and $\delta>0^{5}$ it is quite possible that the negative term in the right-hand side of (25) is subdominant when compared to the positive term. In this case one could obtain $M>0$, thus implying normal attractive gravitational effects for our KR monopole. This is indeed the case in our problem, as we show in the next subsection.

Before doing so, it must be noted that the presence of a de Sitter (positive cosmological constant) space-time in the

\footnotetext{
${ }^{4}$ As can be readily seen from (24), the interior region of radius $\delta$ (Fig. 1) cannot be a flat empty space with zero cosmological constant.

${ }^{5} \delta$ is not necessarily small, as we shall see below.
} 
interior region $0<r<\delta$ implies stability of the resulting KR monopole, as a consequence of a balance between the de-Sitter-induced repulsive forces and the positive-massinduced (normal gravitational) attractive forces on the surface $\delta$. This contrasts with the situation for the original global monopole solution of [2] where there is still an ongoing debate [12] on its stability. We may therefore consider the de Sitter regularization as a necessary physical property of our self-gravitating KR monopole which can guarantee its stability.

\section{B. Regularization scheme: Similar to global monopole case}

To this end, we first require that the regularizing cosmological constant $\Lambda$ in the inner de Sitter space-time coincides with the vacuum (dark) energy of the gravitational Lagrangian (1) in the absence of any other matter fields apart from the scalars $\chi^{A}$ (that is, ignoring KR axions and electromagnetic fields, for reasons stated previously). A vacuum energy $\Lambda$ arises on noting that $\chi^{A} \rightarrow 0$ in the inner de Sitter region, compatible with the small $r$ behavior (16). This interpretation ensures the same regularization process in the cases of both global and KR electromagnetic monopoles. We can then identify [9]:

$$
\Lambda=\frac{1}{4} \lambda \eta^{4}>0, \quad \text { for } \lambda>0 .
$$

The following regime of parameters characterizes the phenomenologically interesting KR electromagnetic monopole of [1], which we now use:

$$
\lambda \gg 1, \quad|\zeta| \gg 1, \quad \lambda \zeta^{2} \gg 1, \quad \eta^{2} \ll 1, \quad \lambda \eta^{2} \ll 1 .
$$

In this regime, we obtain from (25) and (26):

$$
\begin{aligned}
\delta & \sim 2^{3 / 4}|\zeta|^{1 / 2} \lambda^{-1 / 4} \eta^{-1} \\
& =2^{3 / 4} \frac{|\zeta|}{\eta}\left(\zeta^{2} \lambda\right)^{-1 / 4} \gg 1, \\
0<M & =\frac{m}{8 \pi} \sim 0.79|\zeta| \eta\left(\lambda \zeta^{2}\right)^{1 / 4},
\end{aligned}
$$

where we took into account (27) and (7). We thus observe that in this case, both the cutoff $\delta$ and the mass coefficient $M$ are proportional to the KR-axion charge $\zeta$ (or equivalently the magnetic charge of the monopole), and $M$ is positive, implying normal (attractive) gravitational effects, in contrast to the regularized global monopole case [9]. Phenomenologically we are interested in $M \ll 1$, which can be arranged for sufficiently small $0<\eta \ll 1$. Taking into account (27), we observe that the monopole mass is much larger than the $\mathrm{SO}(3)$ spontaneous symmetry breaking scale, $M \gg \eta$.
By identifying

$$
\delta=\alpha L_{c}
$$

we can obtain from (28) and (19) the following estimate for the parameter $0<\alpha<1$ :

$$
0<\alpha=1.26\left(\lambda \zeta^{2}\right)^{-1 / 2} \ll 1,
$$

in view of (27).

Despite the smallness of $\sqrt{\alpha}$, however, both the cutoff $\delta=\alpha L_{c}$ and the shell radius $L_{c}$ are much larger than the Planck scale, consistent with the assumptions and estimates of [1]. Moreover, since $\sqrt{\alpha} \propto \lambda^{-1 / 4}$, ignoring terms of $\mathcal{O}\left(\frac{1}{\lambda}\right)$ for strong coupling $\lambda$ in the estimate (19) is consistent. Thus, although the baglike RN shell (dark shaded region in Fig. 1) is not so thin, nonetheless the most significant contributions to the energy integral (17) come indeed from large radial distances in the integrand, in qualitative agreement to the estimates in [1].

With the value of $\alpha \ll 1$ (30), the total energy (18) becomes to leading order:

$$
\mathcal{E} \simeq 8 \pi\left(1.34\left(\lambda \zeta^{2}\right)^{1 / 4}|\zeta| \eta\right)
$$

where we pulled out explicitly the coefficient $8 \pi$, which facilitates a direct comparison with the monopole mass $m=8 \pi M$ (28). From (31), (28) we obtain for the ratio

$$
\mathcal{E} / m \sim 1.7
$$

implying that, as a result of the divergent nature of the energy due to the angular deficit $\eta^{2}$ in the asymptotic spacetime, the mass coefficient appearing in the gravitational potential is different from the total energy (which in a flat space time would be considered as the total monopole rest mass). This violation of the weak equivalence principle and the invalidity of Birkhoff's theorem (due to the linear dependence of $\mathcal{E}$ on the cutoff $L_{c}$ ) are related. However, the order of magnitude of both terms in the regularized black hole is the same; this is to be expected for large cores, since the space-time in their exterior is practically flat Minkowski (with a small deficit angle, see Fig. 1).

We should remark at this point that for an extended object, such as the regularized monopole, we should be careful to use the "correct type" of mass in the presence of a gravitational field. In the next section we shall elaborate on this issue by defining properly the concept of an "effective mass" for our monopole solution in the presence of the deSitter regularizing core region.

\section{EFFECTIVE MASS CONCEPT IN THE REGULARIZED KR MONOPOLE SPACE-TIME}

We would like to place the above results, especially (32), within the framework of standard concepts of mass in 
general relativity. In [13] it was pointed out that in the (conventional) RN solution to the Einstein field equations, charge, like mass, admits a space-time signature, since it induces curvature of space-time. In view of the proportionality of the charge $\zeta$ to the total mass $M(28)$ or energy $\mathcal{E}$ (31), this is exactly what happens in our self-gravitating KR magnetic monopole case.

In [13] it is argued that a spatial spherical surface of radius $R$, with the singularity of the RN located at the centre of the sphere, and argues that one can define two kinds of "effective mass" consistent with general relativity: one is an effective mass $M_{\text {eff }}^{\text {Int }}$ enclosed by the surface of radius $R$ (that is, associated with the inner region corresponding to radial distances $r<R$ ) and the other is an effective mass $M_{\mathrm{eff}}^{\mathrm{Ext}}$ associated with the exterior region $r>R$, extending up to spatial infinity.

The effective mass contained in a region $r<\delta$ can be calculated using Whittaker's theorem [13,14], according to which

$$
M_{\mathrm{eff}}^{\mathrm{Int}}=\frac{1}{4 \pi} \oint_{v_{2}} d v_{2} V_{, i} n^{i}, \quad n^{i}=(V, 0,0)
$$

where, in spherical polars, $d v_{2}=\delta^{2} \sin \theta d \theta d \phi$ and $V^{2}$ denotes the temporal component of the metric tensor, as defined by the invariant line element $d s^{2}=$ $V^{2} d t^{2}-g_{i j} d x^{i} d x^{j}$.

In the case of the $R N$ metric (in units of the gravitational constant $G=\frac{1}{8 \pi}$ )

$$
V^{2}=1-\frac{2 M}{r}+\frac{Q^{2}}{r^{2}}
$$

with $M$ the Schwarzschild mass (28) and $Q$ the charge, one has

$$
V=\left(1-\frac{2 M}{r}+\frac{Q^{2}}{r^{2}}\right)^{1 / 2}
$$

On substituting in (33) with $\delta=R$, one obtains [13]:

$$
M_{\mathrm{eff}}^{\mathrm{Int}}=M-\frac{Q^{2}}{R}
$$

The effective mass associated with the exterior region $r>R$, has also been calculated in [13] and the result is

$$
M_{\mathrm{eff}}^{\mathrm{Ext}}=\frac{Q^{2}}{R},
$$

so that the sum

$$
M_{\text {eff }}^{\text {tot }} \equiv M_{\text {eff }}^{\mathrm{Int}}+M_{\mathrm{eff}}^{\mathrm{Ext}}=M .
$$

The reader should notice the negative mass contribution in the right-hand-side of (36), which is compensated by the respective positive contribution in the effective mass of the exterior region (37), so that the sum $M_{\text {eff }}^{\text {tot }}$ (38) yields the Schwarzschild mass $M$ of the RN black hole. The effective mass (36) appears [13] in the expression for the radial acceleration of a neutral test particle falling into the RN black hole ( $\tau$ is the proper time):

$$
\frac{d^{2} r}{d \tau^{2}}=-\frac{1}{r^{2}}\left(M-\frac{Q^{2}}{r}\right),
$$

implying that the gravitational field, which in general varies with the distance $r$, becomes repulsive when the effective mass $M-\frac{Q^{2}}{r}$ becomes negative at $r<Q^{2} / M$. Thus, neutral matter falling into the RN black hole will ultimately accumulate on a $(2+1)$-dimensional hypersurface for which the effective mass $M-\frac{Q^{2}}{r}=0$.

One may then attempt to apply the above considerations of [13] on this surface, by defining appropriately the two types of effective mass discussed previously. However, there are important differences. First, in view of the deficit angle $\eta^{2}$, the total energy in the exterior region is divergent formally and we cut such a divergence off using a bag model, and second, the inner region enclosed by the spherical surface of radius $\delta(25)$ is described by a different (de Sitter) space-time, which acts as a regulator of the RN singularity. Nonetheless, there are some features of our regularized solution that are in qualitative agreement with the RN analysis of [13] as far as the geometrical role of the charge is concerned. Let us see what implications such considerations have on our understanding of relations like (32) that we have found above to characterize our solution.

In our problem, in the exterior region we do have a $\mathrm{RN}$ like geometry (5), with the (magnetic monopole) charge $Q^{2}$ being sourced by the KR axion charge:

$$
Q^{2}=2 \zeta^{2}=g^{2}
$$

on account of (11). In the context of our baglike model of the monopole [1], the total energy (31) when expressed in terms of $\delta$ (28), using (40), yields

$$
\mathcal{E} \simeq 1.13 \frac{Q^{2}}{\delta} .
$$

The effective mass contained in the region $r<\delta$ can be calculated using Whittaker's theorem [13,14](33), using the de Sitter metric (22), for which

$$
V=\left(1-\frac{1}{3} \Lambda r^{2}\right)^{1 / 2},
$$

with $\Lambda$ given in (26). Then, from (33) we obtain a negative effective mass (consistent with the repulsive gravitational nature of de Sitter space time) 


$$
M_{\mathrm{eff}}^{\mathrm{Int}}=-\frac{1}{3} \Lambda \delta^{3}<0
$$

If one requires (as in the $\mathrm{RN}$ case) that the sum of both exterior and interior effective masses should equal the Schwarzschild mass [(38)] then in our case one should have

$$
M_{\mathrm{eff}}^{\mathrm{Ext}}=\frac{Q^{2}}{\delta}
$$

which is consistent with (37) for $R=\delta$. From (28), (43), and (44), one can verify the validity of (38) since

$$
M_{\mathrm{eff}}^{\mathrm{Ext}}=\frac{3}{2} M \quad \text { and } \quad M_{\mathrm{eff}}^{\mathrm{Int}}=-\frac{1}{2} M .
$$

From (41) and (44) we observe that we obtain a value for the total energy $\mathcal{E}$ in the shell slightly larger than the effective mass $M_{\text {eff }}^{\text {Ext }}$ in the region exterior to the core. ${ }^{6}$ This can be attributed to the nonzero contributions of the $\mathrm{KR}$ axion $b(x)$ and electromagnetic fields to the energy functional $\mathcal{E}(31)$, as well as the gravitational self-binding energy. Generically, in nearly flat space-times, as is the case in the exterior region of the core, one can define the total energy of an extended object as the integral of the temporal component of the stress tensor over a spatial volume $E_{\text {total }}=\int d V T_{00}$. Under the assumptions that the components of the total momentum vector of the system are zero and the object is considered "quasistatic," that is, there is no significant energy present in the form of gravitational waves, then the object's "mass" $\tilde{m}$ can be defined as (in units of the speed of light in vacuo):

$$
\tilde{m}=E_{\text {total }}+E_{\text {binding }},
$$

where $E_{\text {binding }}<0$ denotes the Newtonian gravitational self-binding energy. In our case, for large core radius, the criterion of (approximate) space-time flatness, along with the other assumptions, is satisfied; one may thus identify $E_{\text {total }}=\mathcal{E}$ and $\tilde{m}=M_{\text {eff }}^{\text {Ext }}$ in (46). Hence, the fact that the total energy is larger than the mass is naturally explained.

We also note that, for us, the role of the infalling neutral matter is played by the KR axion pseudoscalar field, which thus will accumulate on the surface of radius $\delta$, since it is on this surface that the radial acceleration will vanish (39).

\footnotetext{
${ }^{6}$ The reader should recall that the total energy (41), in terms of the Schwarzschild mass (28), is given by (32) [which also stems from (41), (44), and (45)]. In our discussion of the weak equivalence principle in this section, we relate the shell energy $\mathcal{E}$ to the exterior effective mass $M_{\text {eff }}^{\text {Ext }}$, rather than the Schwarzschild mass $M$, since the latter contains contributions [cf. (38)] from both the (de Sitter) core and exterior regions of the selfgravitating KR monopole.
}

We can summarize that, in view of the negative effective mass contributions of the de Sitter regulator, the weak equivalence principle, where one would equate the total energy $\mathcal{E}$ with the inertial mass, fails. However, this should be expected for gravitating extended objects, as is our case, given that the weak equivalence principle characterizes pointlike masses. Nevertheless, since the core radius is very large (compared to the Planck length), it is expected that such discrepancies will be small, since gravitational effects in the exterior of the core would be suppressed. This is indeed the situation that characterizes our case, where the gravitational total energy (41) is found to be almost the same magnitude as the effective mass of the monopole (44). From the approximate validity of the weak equivalence principle, we can conclude that the motion of our monopole is akin to a point particle.

It is interesting to note, though, that the weakequivalence principle can be accommodated exactly through a choice of the value of our regularizing cosmological constant $\Lambda$ in the core region, such that

$$
\frac{\mathcal{E}}{M_{\mathrm{eff}}^{\mathrm{Ext}}}=1, \quad M_{\mathrm{eff}}^{\mathrm{Ext}}=\frac{Q^{2}}{\delta} .
$$

In this scheme, the positive contributions to the total energy functional from the cosmological constant, KR axion, and electromagnetic fields will screen the negative binding energy due to the gravitational effects [cf. (46)], leading to (47). This choice of regularization scheme is consistent with a negative dilaton potential in the de Sitter region, where the string theory [the ultraviolet (UV) completion of our low energy model] is strongly coupled and such a potential might be generated, for example, through nonperturbative string-loop corrections. Outside this region, where string theory is weakly coupled and our low energy model is an effective description of the dynamics, the tree level dilaton potential vanishes due to arguments based on conformal invariance [3]. In this way, our asymptotic solutions (which are valid outside the de Sitter region) are not affected. Consequently we can introduce, instead of (26), a regularized $\Lambda^{\xi}$ :

$$
\Lambda^{\xi}=\frac{1}{4} \xi^{2} \lambda \eta^{4}>0, \quad \text { for } \lambda>0
$$

with $\xi \in \mathrm{R}$ a real number to be determined. We obtain from (25) and (27):

$$
\begin{aligned}
\delta & \simeq 2^{3 / 4}|\xi| \frac{|\zeta|}{\eta}\left(\zeta^{2} \lambda\right)^{-1 / 4} \\
m^{\xi} & \simeq 8 \pi\left(\frac{2^{1 / 4}}{|\xi|}-\frac{1}{6} 2^{5 / 4}|\xi|^{5}\right)|\zeta|\left(\lambda \zeta^{2}\right)^{1 / 4} \eta
\end{aligned}
$$

and from (29) we also obtain 


$$
0<\alpha=1.26 \xi^{2}\left(\lambda \zeta^{2}\right)^{-1 / 2} \ll 1,
$$

since we expect $|\xi|=\mathcal{O}(1)$, given that the role of $\xi$ is to set the ratio in (32) to one. On account of (48), we obtain for the energy (18):

$$
\mathcal{E}^{\xi} \simeq 1.13\left|\xi^{2}\right| \frac{Q^{2}}{\delta}
$$

instead of (41). Hence, requiring

$$
\xi^{2}=\frac{1}{1.13} \simeq 0.89
$$

we ensure (47). This implies a smaller cosmological constant than previously chosen (26).

\section{CONCLUSIONS}

In this paper we have regularized the curvature singularity characterizing our recent self-gravitating KR electromagnetic monopole solution [1]. The regularization was achieved by cutting off the singular region by means of a de Sitter space implied by the scalar sector of the theory. The pertinent positive cosmological constant was proportional to the fourth power of the spontaneous symmetry breaking scale $\eta$ of the internal $\mathrm{SO}(3)$ symmetry of the model. We employed Israel-junction conditions when matching this interior region with the Reisssner-Norström (RN) blackhole space-time that characterizes the outer region. This regularization and our earlier bag-like structure [1] allows, in a self-consistent way, for a calculation of both the finite total energy $\mathcal{E}$ of the monopole, and the mass coefficient $m$ appearing in the appropriate gravitational potential term of the RN metric. Notably in this case, $m$ cannot be identified necessarily with the total energy, due to the divergent infrared behavior of the latter. This feature also characterizes the global monopole case [2], in the absence of the KR charge. However, in this latter case, the above matching leads to a negative gravitational mass coefficient $m<0$ and thus repulsive gravitational effects [9].

By contrast, in our KR electromagnetic monopole case, the gravitational mass coefficient $m$ turns out to be positive, but proportional to the KR axion charge, whose role is thus crucial in ensuring normal (attractive) gravitational effects of the KR monopole. The pertinent calculations of the total energy $\mathcal{E}$ of the monopole and $m$ have been performed within a phenomenologically interesting regime of the parameters of the model, in which the scale $\eta$ is assumed much smaller than the Planck scale, since our interest is to consider monopoles with masses within the range of current or future colliders. When employing a regularization using a de Sitter space-time region with the value of the cosmological constant used in the global monopole case, we find that $\mathcal{E} \neq m$. (Although both quantities in the ratio are of the same order of magnitude, with values very close to each other.) This small violation of the weak equivalence principle is to be expected since we deal in our case with gravitational effects that have nontrivial contributions to the effective mass. We put our result into context by comparing with studies of conventional RN solutions [13]; we can obtain agreement between the two approaches, in the sense of (38), by choosing a cosmological constant which is slightly reduced in this case as compared to that of the global monopole case [9]. We have speculated that, from a microscopic view point, such a regularization might arise from a nontrivial dilaton dynamics inside the core de Sitter region, giving rise to a negative dilaton potential in that region. Outside the region, the dilaton is stabilized to a constant value, corresponding to a zero value of its potential, and thus the asymptotic solutions of Ref. [1] are not affected.

We reiterate that, the interpretation of the de Sitter space regularization of our monopole core as a repulsive gravitational force, allows us to understand the stability of the self-gravitating KR solution; the stability is a consequence of a balance of these repulsive forces with the attractive gravitational forces of the positive mass parameter of the $\mathrm{RN}$ space-time in the exterior region, at the radius $\delta$ where the Israel matching conditions of the two metrics are enforced. The nontrivial KR-axion charge $\zeta$ of our model is crucial to produce this balance.

By considering, in detail, phenomenologically realistic microscopic scenarios for the KR monopole it would be possible to determine the symmetry breaking scale $\eta$ from first principles, and thus make definite predictions for the monopole mass. Moreover, the role of the various fields, like the scalars $\chi^{A}$, would also be elucidated, and this will lead to a better understanding of the production mechanism of such monopoles. Since the KR electromagnetic monopoles are composite, nonpointlike, objects, their production at colliders is expected to be strongly suppressed, according to generic arguments [15]. However, non-suppressed production may be expected for such objects in environments with high temperature and/or strong external magnetic fields, as a consequence of a thermal Schwinger-like mechanism, according to recent arguments [16]. Hence, the demonstration from first principles of how a low mass KR monopole arises in the physical spectrum of microscopic models, is a pressing issue, not only of theoretical, but also of direct experimental relevance. We hope to attempt to answer (some of) the above questions in the future.

\section{ACKNOWLEDGMENTS}

The work of N. E. M. and S. S. is partially supported by Science and Technology Facilities Council (UK) under the research Grant No. ST/P000258/1. 
[1] N. E. Mavromatos and S. Sarkar, Magnetic monopoles from global monopoles in the presence of a Kalb-Ramond field, Phys. Rev. D 95, 104025 (2017).

[2] M. Barriola and A. Vilenkin, Gravitational Field of a Global Monopole, Phys. Rev. Lett. 63, 341 (1989).

[3] D. J. Gross and J.H. Sloan, The quartic effective action for the heterotic string, Nucl. Phys. B291, 41 (1987); R. R. Metsaev and A. A. Tseytlin, Order alpha-prime (two loop) equivalence of the string equations of motion and the sigma model Weyl invariance conditions: Dependence on the dilaton and the antisymmetric tensor, Nucl. Phys. B293, 385 (1987); M. J. Duncan, N. Kaloper, and K. A. Olive, Axion hair and dynamical torsion from anomalies, Nucl. Phys. B387, 215 (1992); R. T. Hammond, Torsion gravity, Rep. Prog. Phys. 65, 599 (2002).

[4] B. Zwiebach, A First Course in String Theory (Cambridge University Press, Cambridge, England, 2009), p. 673.

[5] I. Antoniadis, C. Bachas, J. R. Ellis, and D. V. Nanopoulos, Cosmological string theories and discrete inflation, Phys. Lett. B 211, 393 (1988); An expanding universe in string theory, Nucl. Phys. B328, 117 (1989).

[6] H. Reissner, Über die Eigengravitation des elektrischen Feldes nach der Einsteinschen Theorie, Ann. Phys. (Berlin) 355, 106 (1916); G. Nordström, Verhandl. Koninkl. Ned. Akad. Wetenschap., Afdel. Natuurk., Amsterdam 26, 1201 (1918).

[7] W. Israel, Singular hypersurfaces and thin shells in general relativity, Nuovo Cimento B 44, 1 (1966); Erratum, Nuovo Cimento B 48, 463 (1967).

[8] O. B. Zaslavskii, Classical model of elementary particle with Bertotti-Robinson core and extremal black holes, Phys. Rev. D 70, 104017 (2004).

[9] D. Harari and C. Lousto, Repulsive gravitational effects of global monopoles, Phys. Rev. D 42, 2626 (1990).

[10] S. H. Mazharimousavi and M. Halilsoy, Ternary cubic forms having bounded invariants, and the existence of a positive proportion of elliptic curves having rank 0, arXiv:1703 .05286v2, and references therein.

[11] F. R. Klinkhamer, Black-hole solution without curvature singularity, Mod. Phys. Lett. A 28, 1350136 (2013); Blackhole solution without curvature singularity and closed timelike curves, Acta Phys. Polon. B 45, 5 (2014); A new type of nonsingular black-hole solution in general relativity, Mod. Phys. Lett. A 29, 1430018 (2014); F. R. Klinkhamer and C. Rahmede, A nonsingular spacetime defect, Phys. Rev. D 89, 084064 (2014).

[12] A. S. Goldhaber, Collapse of a 'Global Monopole', Phys. Rev. Lett. 63, 2158 (1989); In the original suggestion of Goldhaber that global monopoles are not stable against "angular" collapse, there is an ongoing debate on this issue; for a partial list of references see: S. H. Rhie and D. P. Bennett, Global Monopoles Do Not 'Collapse', Phys. Rev. Lett. 67, 1173 (1991); L. Perivolaropoulos, Instabilities and interactions of global topological defects, Nucl. Phys. B375, 665 (1992); G. W. Gibbons, M. E. Ortiz, F. Ruiz Ruiz, and T.M. Samols, Semilocal strings and monopoles, Nucl. Phys. B385, 127 (1992); M. Hindmarsh, Semilocal topological defects, Nucl. Phys. B392, 461 (1993); G. Arreaga, I. Cho, and J. Guven, Stability of self-gravitating magnetic monopoles, Phys. Rev. D 62, 043520 (2000); A. Achucarro and J. Urrestilla, The (In) stability of Global Monopoles Revisited, Phys. Rev. Lett. 85, 3091 (2000); R. Gregory and C. Santos, Space-time structure of the global vortex, Classical Quantum Gravity 20, 21 (2003); E. R. Bezerra de Mello, Reply on comment on 'Gravitating magnetic monopole in the global monopole space-time', Phys. Rev. D 68, 088702 (2003); S. B. Gudnason and J. Evslin, Global monopoles of charge 2, Phys. Rev. D 92, 045044 (2015).

[13] G. E. Marsh, Charge, geometry, and effective mass, Found. Phys. 38, 293 (2008).

[14] E. T. Whittaker, On Gauss' theorem and the concept of mass in general relativity, Proc. R. Soc. A 149, 384 (1935).

[15] A. K. Drukier and S. Nussinov, Monopole Pair Creation in Energetic Collisions: Is It Possible?, Phys. Rev. Lett. 49, 102 (1982).

[16] O. Gould and A. Rajantie, Thermal Schwinger pair production at arbitrary coupling, Phys. Rev. D 96, 076002 (2017); Magnetic Monopole Mass Bounds from Heavy Ion Collisions and Neutron Stars, Phys. Rev. Lett. 119, 241601 (2017). 